\section{FOREIGN BODY IN THE BRONCHIAL TUBE.}

\section{By Frederick Page, Esq., Perth, Western} Australia.

A. F., a child, aged 3 years, was seized on November 12th, 1861, with violent and long continued cough, threat. ening death by suffocation. These symptoms were sup. posed to be caused by a piece of slate-pencil having slipped from the child's mouth through the rima of the glottis into the trachea. Next day there was active pneumonia on both sides of the chest, from which the little patient recovered after an illness of three weeks. From this time up to December 6th, 1862, the child's health was bad, owing to repeated attacks of bronchitis, diarrhoa, and irritability of stomach. On the evening of December 6th, after a violent fit of coughing, the child became black in the face, was unable to breathe, and died immediately.

A post mortem examination showed that both lungs were diseased. The left had formed adhesions all round the chest, and was carnified. In the left bronchial tube a piece of slate-pencil, an inch in length, was found : it had no doubt caused death, by being suddenly coughed into the rima of the glottis, whence it had re-fallen into its old position as soon as the muscular spasm went off at the time, or immediately after death.

This case is interesting on account of the size of the foreign body, the age of the child, and from the circum. stance that it was found in the left bronchus instead of the right, as is most commonly the case.

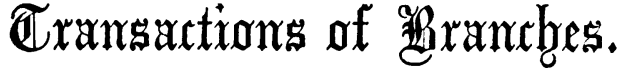

SHROPSHIRE SCIENTIFIC BRANCH.

THE PRESENT TREATMENT OF DISEASE.

By Wr. Newman, M.D.Lond., St. Martin's, Stamford.

[Read February 13th, 1863.]

Medicine is in its very essence a science of progress discoveries in closely allied branches of scientific inquiry have had more or less marked influence on the Medical Art; and hence bave resulted changes, both in theory and practice, too numerous to classify or even refer to.

The ars mirabilis has been reproached with this attribute as though it were a material infirmity, and in most respects with but insufficient reason. The very conditions of an exact science are wanting; the postulates and definitions are not clearly laid down; and, though the field of inquiry be boundless, and its interest inexhaustible, the landmarks even are undetermined. It is true, that the art and science of medicine is dependent on facts; but these facts are not only few in number, and widely scattered over an extensive area of observation, but they present also only very slender links of connexion between themselves.

The observer is naturally impatient of results, draws his deductions from materials imperfectly arranged, viti. ated, it may even be, by faulty lines of research, and thus forms structures of theory, brilliant in seeming, lasting for some short time, yet lacking a definite or reliable basis.

Some of the most interesting volumes that have of late issued from the medical press, direct our attention to the differences of medical treatment, past and present. The limits of this paper will allow but a hasty glance at some items of comparison between the two great schools; yet I would venture to hope that so short a notice may not be without its advantages.

To take one class of diseases to exemplify the purport of this paper-Acute or Inflammatory Diseases. By the older physicians, inflammation was looked upon as a something to be fought with and overcome-as a morbid something seizing on a portion of the human frame, and only to be combated with success by the employment of active treatment. The antiphlogistic plan (borrowing its title from a chemical doctrine long since passed away) was put in practice in all its vigour.

The abstraction of blood was the first and principal remedy : general, by opening artery or vein; local, by use of scarificator and cupping-glass, or by application of leeches. This procedure was resorted to in every instance where pain, fever, and impeded function coexisted, in the first place with the avowed intention of cutting short the disease, or at least of moderating the violence of its attack by diminishing both the force and the frequency of the circulation. Failing this result, the same remedy was employed in milder form to remove the consequences of the inflammatory invasion, and thus to aid in restoring the affected part to its normal condition.

The next point was the Adoption of Low Diet. Watery or farinaceous compounds were alone allowed; animal food in any form and alcobolic stimulants being abso. lutely forbidden.

In the Administration of Depressing Remedics, mercurials should certainly take the first place, as most varied in form, most active in character, and decisive in effect. They have been usually administered in small and frequently repeated doses, combined with opium to ensure retention in the system, and continued either until the symptoms were materially relieved, or until the constitutional effects of the mineral were manifested by salivation more or less distinct.

Antimonials have been deemed of especial value in thoracic inflammations, and employed first by Rasori, an Italian physician, in large and repeated doses, in the treatment of pneumonia. We are told that, in this particular affection, there exists on the part of the system an extreme tolerance of this form of remedial agent; and that its usual consequences, vomiting and purging, do not occur until the morbid action is yielding.

The list is by no means ended. The mineral and vegetable kingdoms were each invoked: digitalis; colchicum; neutral salts; purgatives more or less active; medicines acting specially on the skin and kidneys-all had their place as aids in the active treatment of inflammatory disease.

By the majority, however, of those who follow in the steps of the authorities of the earlier part of this century, the antiphlogistic treatment, though still practised in acute affections, is not resorted to in the same unsparing manner. Direct depletion is no longer the constant rule; yet the principles of their belief are the same, and the weapons on which they mostly rely are of depressing character.

The later men, and preeminent should be named Dr. Hughes Bennett of Edinburgh, have taken a far different course in this class of affections. The view they advocate is, that inflammation owes its origin to the presence of some agent in the system, in itself depressing; and that the attack, per se, is proof of deterioration of vital power, and hence to be best treated, not by means that still further reduce that power, but by careful avoidance of hurtful measures, and by the use of means which will uphold the system and favour the removal of the poison by the natural emunctories-in one word, by a supporting and eliminating treatment.

The mode of treatment suggested in conformity with these views is, briefly, the observance of extreme quietude; the administration, neither sparing nor unfrequent, of easily assimilable nutriment, such as beef-tea, etc.; with the administration of such medicines as shall favour the excretion from the system of the results of morbid action.

The members of a more adranced school of modern physicians, among whom should be specially named the 